\title{
UNIFORMIDADE DE DISTRIBUIÇÃO DE LÍQUIDO E DE AR AO LONGO DA BARRA DE PULVERIZAÇÃO
}

Murilo Mesquita Baesso ${ }^{1}$, Mauri Martins Teixeira², Rogério Faria Vieira ${ }^{3}$, Trazilbo José de Paula Junior ${ }^{4}$, Paulo Roberto Cecon $^{5}$

\section{RESUMO}

O desenvolvimento de novas tecnologias de aplicação de fitossanitários visa à redução da contaminação ambiental, da deriva, do volume de calda com aumento da segurança do aplicador e melhoria da deposição da calda, para melhorar a qualidade da pulverização. O objetivo deste trabalho foi avaliar a uniformidade de distribuição de ar ao longo da barra de pulverização com assistência de ar, bem como avaliar a uniformidade de distribuição de líquido de bicos de pulverização hidráulica de jato cônico vazio, modelo JA-4, marca Jacto, além de bicos de jato leque, modelo AXI-110-04, marca Jacto, trabalhando com quatro velocidades de rotação do ventilador e três alturas da barra de pulverização em relação à bancada de ensaios. A rotação do eixo do ventilador que apresentou os melhores resultados foi a de $2.100 \mathrm{rpm}$. Os perfis de distribuição do ar foram irregulares, e as distribuições do vento a 0,5 $\mathrm{m}$ da barra apresentaram coeficientes de variação menores que as obtidas quando a barra estava a $0,3 \mathrm{~m}$ do ponto de leitura.

Palavras-chave: Fitossanitários, qualidade de aplicação e deposição de calda.

\section{UNIFORMITY OF LIQUID AND AIR DISTRIBUTION ALONG THE SPRAY BAR}

\begin{abstract}
The development of new technologies of phytosanitary applications aims to reduce environmental contamination, drift, liquid volume and increase the safety of the applier, as well as the improvement of the liquid deposition, in order to improve the spraying quality. The objective of this work was to determine the uniformity of air distribution along the airassisted spraying bar, and evaluate the uniformity of the liquid, carried out with four speeds of fan rotation and three heights of the spray bar in relation to the test bench. The fan axis rotation of $2.100 \mathrm{rpm}$ presented the best results. The air distribution profiles were irregular, and the wind distribution at $0.5 \mathrm{~m}$ from the bar presented a variation coefficient lower than that obtained when the bar was $0.3 \mathrm{~m}$ far from the reading point.
\end{abstract}

Keywords: Phytosanitary, quality of application and liquid deposition.

\section{Recebido para publicação em 06/05/2009. Aprovado em 11/11/2009}

1-Eng ${ }^{\circ}$ Agrônomo, D.Sc., Departamento de Engenharia Agrícola, Viçosa-MG, CEP: 36570-000, e-mail:murilo.baesso@ufv.br

2-Eng ${ }^{\circ}$ Agrônomo, Prof. Doutor, Departamento de Engenharia Agrícola, Viçosa-MG, CEP: 36570-000, e-mail: mauri@ufv.br

3-Eng ${ }^{\circ}$ Agrônomo, D.Sc , Empresa de Pesquisa Agropecuária de Minas Gerais, Viçosa-MG, CEP: 36570-000, e-mail: rfvieira@epamig.br

4-Eng ${ }^{\circ}$ Agrônomo, D.Sc, Empresa de Pesquisa Agropecuária de Minas Gerais, Viçosa-MG, CEP: 36570-000, e-mail: trazilbo@epamig.br

5-Eng Agrônomo, Prof. Doutor, Departamento de Informática, Viçosa-MG, CEP: 36570-000, e-mail: cecon@dpi.ufv.br 


\section{INTRODUÇÃO}

A eficácia de um tratamento fitossanitário está relacionada a vários fatores, como escolha do produto, tipo de bico, pressão de trabalho, volume de calda aplicado, cobertura do alvo e escolha correta do equipamento de aplicação (MATTHEWES, 2004). É necessária uma técnica de aplicação específica para cada praga a ser controlada. No caso do fungo Sclerotinia sclerotiorum, há a preocupação de que a calda alcance as partes inferiores da planta, pois para ocorrer a infecção primária o fungo necessita de fonte de energia exógena, geralmente as pétalas de flores.

Entre as técnicas de aplicação de fungicidas para o controle do mofo-branco, destacam-se a que se baseia na pulverização hidráulica do produto e a aplicação de fungicidas via água de irrigação, denominada fungigação. As aplicações aéreas tendem a não proporcionar bons resultados de controle do mofo-branco, já que, em razão do baixo volume de calda utilizado por esse sistema, a distribuição do ingrediente ativo no dossel da planta não é uniforme.

Os produtos fitossanitários devem ser aplicados com máxima eficiência, sendo necessário estudar a deposição, a cobertura e a deriva das gotas durante a pulverização. Entre outros fatores, a deriva é responsável por perdas do produto, além de contribuir para a contaminação ambiental. Para que o fitossanitário seja aplicado de forma eficiente e atinja o resultado esperado, é necessária uma cobertura adequada em todas as partes da planta (MATTHEWES, 2000).

Os bicos de pulverização proporcionam perfil característico que depende da vazão nominal, do ângulo formado pelo jato, da altura da barra portabicos e da qualidade de fabricação. Esse perfil deve ser considerado nas recomendações de utilização dos bicos, de maneira a obter distribuição uniforme. Uma distribuição uniforme é caracterizada por um baixo coeficiente de variação da distribuição volumétrica de líquido, tanto no sentido longitudinal quanto no transversal. Em aplicações em área total, a uniformidade transversal depende do bico utilizado, da sobreposição dos jatos e da posição da barra porta-bicos em relação ao plano de tratamento (BARTHELEMY et al., 1990).

O desenvolvimento de equipamentos eficientes de aplicação é um dos objetivos das pesquisas com aplicação de fitossanitários. Segundo Teixeira et al. (2008), faz-se necessário o estudo de técnicas mais acuradas, com consequente melhoria na uniformidade de deposição do produto no alvo.

Equipamentos com assistência de ar têm sido usados para aumentar a penetração das gotas no dossel da cultura e reduzir a deriva das pulverizações (BAUER \& RAETANO, 2000). Os pulverizadores hidráulicos tratorizados com assistência de ar na barra porta-bicos possuem um ou dois ventiladores, geralmente axiais, posicionados em local próximo à seção central da barra de pulverização, os quais distribuem volume muito grande de ar no interior de um duto inflado montado acima da barra portabicos (MATTHEWS, 2000). Segundo Andersen et al. (2000), essas características melhoram a eficácia sem aumentar a quantidade de calda.

Bauer e Raetano (2000) avaliaram o efeito da assistência de ar na deposição e perdas de produtos fitossanitários em pulverizações da soja em relação ao equipamento convencional, com alvos artificiais. Constataram níveis significativamente menores de deriva aerotransportada com o equipamento dotado de assistência de ar, em diferentes alturas da barra pulverizadora. Encontraram também menor deriva por sedimentação na área externa da cultura com o uso da assistência de ar na barra pulverizadora.

Segundo a norma do Comitê Europeu de Normalização (prEN 12761-2, ECS,1997), a uniformidade de distribuição medida em termos de coeficiente de variação da distribuição volumétrica (CV) deve situar-se abaixo de 7\% quando o equipamento trabalha nas condições para as quais foi projetado e de $9 \%$ para trabalhos fora dessas condições.

Os objetivos deste trabalho foram determinar a uniformidade de distribuição de ar ao longo da barra de pulverização com assistência de ar, avaliar a uniformidade de distribuição de líquido da pulverização com assistência de ar na barra portabicos e determinar a melhor altura de trabalho da barra porta-bicos.

\section{MATERIAL E MÉTODOS}

Os ensaios foram realizados no Laboratório de Mecanização Agrícola do Departamento de Engenharia Agrícola da Universidade Federal de Viçosa, na cidade de Viçosa, localizada a uma altitude de aproximadamente $650 \mathrm{~m}$, com coordenadas geográficas de $20^{\circ} 45^{\prime} 54$ " Latitude Sul 
e $45^{\circ} 52^{\prime} 54^{\prime \prime}$ de Longitude Oeste. Foi utilizado um pulverizador hidráulico, modelo Falcon Vortex, marca Jacto, com tanque de $600 \mathrm{~L}$ e barra de pulverização com assistência de ar de $14 \mathrm{~m}$ de comprimento. Para acionar e tracionar o pulverizador, foi utilizado um trator Valtra 800 , com $62,5 \mathrm{~kW}$ (85 cv) de potência nominal no motor.

Para determinar a uniformidade de distribuição de ar ao longo da barra do pulverizador, foi considerado o efeito das velocidades do ar na saída do difusor, correspondentes às rotações do eixo axial do ventilador de $1.800 ; 2.100$ e $2.800 \mathrm{rpm}$, com cinco repetições. Foi verificada a velocidade do ar após a sua saída do duto difusor. As posições de verificação da velocidade da cortina de ar localizavam-se em um plano situado a 0,3 e 0,5 m, abaixo da barra de pulverização (Figura 1). As leituras foram efetuadas ao longo da barra de pulverização a cada $0,25 \mathrm{~m}$, ressaltando-se que abaixo de cada bico de pulverização coincidiu uma leitura.

A velocidade do ar foi medida com um termo anemômetro de fio quente fixado em um tripé mantido em nível. A temperatura e umidade relativa do ambiente foram monitoradas com um psicrômetro. As medições foram realizadas em um galpão fechado, para evitar a interferência da corrente do ar externo.

Para a análise da uniformidade da velocidade do ar, as médias das velocidades registradas ao longo da barra foram utilizadas para o cálculo do coeficiente de variação $(\mathrm{CV})$, calculado por:

$$
C V=\left(\frac{\sqrt{\frac{\sum_{i=1}^{n}\left(X_{i}-\bar{X}\right)^{2}}{n-1}}}{\frac{\sum_{i=1}^{n} \cdot X_{i}}{n}}\right) \cdot 100
$$

em que,

$\mathrm{CV}=$ coeficiente de variação;

$\underline{n}=$ número de observações;

$\bar{X}=$ média; e

$\bar{X}_{i}=$ valor observado.

Para determinar a uniformidade de distribuição do líquido ao longo da barra, avaliaram-se pontas de pulverização hidráulica, de jato cônico vazio, modelo JA-4 da marca Jacto, com pressão de operação de 400,600 e $800 \mathrm{kPa}$ e pontas de jato leque, modelo AXI-110-04 da marca Jacto, com pressão de operação de 100, 200 e $300 \mathrm{kPa}$. Previamente, a vazão de líquido das pontas foram determinadas nas pressões estudadas, sendo descartadas as pontas cujas vazões diferiram de $1 \%$ da indicada pelo fabricante. Além disso, verificou-se o ângulo de abertura do jato, feito por meio de imagens frontais dos bicos de pulverização obtidas com o uso de uma câmera digital, marca Nikon, modelo Coolpix, com resolução de 5.1 megapixel. As imagens foram analisadas no programa computacional "Image Tool", versão 3.0. O ângulo de abertura foi medido nas projeções delimitadas tangencialmente às bordas do jato. Para o estudo da simetria do jato, foi medido o ângulo em duas partes em relação ao plano vertical: esquerdo e direito, sendo descartadas os bicos assimétricas.

Foi montado, para cada tipo de ponta, um experimento disposto em esquema fatorial $4 \times 3$, em um delineamento inteiramente casualizado, com três repetições. Os fatores foram: sem assistência de ar (0 rpm) e assistência de ar na barra correspondentes a rotação no ventilador de $1.800,2.100$ e $2.800 \mathrm{rpm}$ e três pressões do líquido $(400,600$ e $800 \mathrm{kPa}$ para o bico de jato cônico vazio e 100, 200 e $300 \mathrm{kPa}$ para o jato leque). Os ensaios foram realizados em três alturas da barra em relação à bancada de distribuição $(0,3 ; 0,4$; e $0,5 \mathrm{~m}$ para o bico de jato cônico vazio
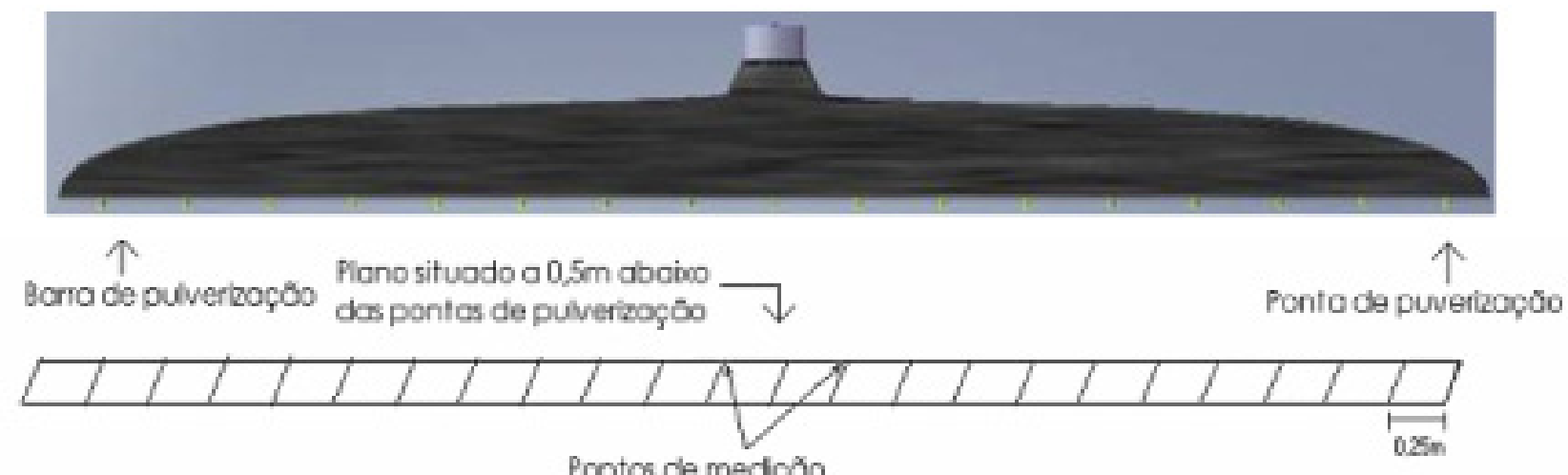

Pontos de mediço

da velocidade do or

Figura 1. Esquema das medições da velocidade do ar produzido pelo ventilador. 
e 0,$4 ; 0,5$; e $0,6 \mathrm{~m}$ para o de jato leque), sendo os espaços entre bicos de $0,5 \mathrm{~m}$.

Foram avaliadas, conjuntamente, cinco pontas ao longo da barra de pulverização, montadas no próprio pulverizador sob uma bancada de ensaios padronizada e construída de acordo com a norma ISO 5682/1 (ISO, 1986).

Durante $60 \mathrm{~s}$, foi recolhido o líquido em provetas ao longo da faixa de deposição dos bicos e registrados os dados para caracterizar a distribuição da pulverização.

Os ensaios foram feitos em galpão coberto e fechado, para minimizar o efeito das condições ambientais. Os manômetros utilizados foram previamente calibrados por meio de um manômetro padrão, obtendo-se a relação entre pressão indicada e pressão real. A temperatura e a umidade relativa do ambiente foram monitoradas com um psicrômetro. A velocidade angular do eixo do ventilador foi medida com um tacômetro digital, marca Tako, modelo TD 303.

$\mathrm{Na}$ avaliação da uniformidade de distribuição volumétrica do conjunto de bicos, os volumes do líquido recolhidos nas provetas alinhadas com as canaletas ao longo da faixa de deposição foram transformados em porcentagem do volume total recolhido para eliminar o fator tempo dos dados analisados. Foi empregado, para avaliação da uniformidade de distribuição, o coeficiente de variação $(\mathrm{CV})$, calculado segundo a Equação 1.
Para os dados de uniformidade de distribuição volumétrica do conjunto de bicos, utilizou-se a metodologia da superfície de resposta. Os modelos foram escolhidos baseados na significância dos coeficientes de regressão utilizando o teste "t" e no coeficiente de determinação.

\section{RESULTADOS E DISCUSSÃO}

\section{Uniformidade de distribuição do ar ao longo da barra de pulverização}

A interação entre rotações do ventilador e alturas da barra porta-bicos não foi significativa, porém tanto as rotações quanto as alturas influenciaram o coeficiente de variação da distribuição de vento ao longo da barra (Quadro 1).

Foi encontrada a seguinte relação entre as médias do coeficiente de variação (CV) da velocidade do vento em função das rotações do ventilador (rv):

$$
\begin{aligned}
& C V(\%)=45,3687-0,0038531^{*} r v \\
& \mathrm{r}^{2}=0,9831
\end{aligned}
$$

*Significativo a $5 \%$ de probabilidade.

Menor coeficiente de variação foi obtido com incremento na rotação do ventilador. Os valores estimados variaram entre 38,43 e $34,58 \%$, trabalhando com 1.800 e 2.800 rpm, respectivamente. Pode-se observar, também, que os resultados a $0,5 \mathrm{~m}$ da barra

Quadro 1. Resumo da análise de variância do coeficiente de variação, em função da rotação do ventilador e da altura da barra porta-bicos

\begin{tabular}{ccc}
\hline FV & GL & QM \\
\hline Altura & 1 & $72,1357^{* *}$ \\
Rotação & 2 & $39,6811^{* *}$ \\
Altura X Rotação & 2 & $3,6864^{\text {ns }}$ \\
Resíduo & 20 & 4,2332 \\
\hline CV $(\%)$ & & 5,59 \\
\hline
\end{tabular}

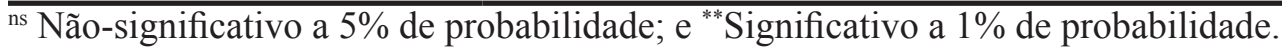

Quadro 2. Coeficiente de variação (CV) médio (\%) da velocidade do vento, em função das distâncias da barra porta-bicos

\begin{tabular}{cc}
\hline Distância $(\mathrm{m})$ & $\mathrm{CV}(\%)$ \\
\hline 0,3 & $38,32^{\mathrm{a}}$ \\
0,5 & $35,22^{\mathrm{b}}$ \\
\hline
\end{tabular}

* Médias seguidas pela mesma letra na coluna não diferem a 1\% de probabilidade, pelo teste $\mathrm{F}$. 
obtiveram coeficiente de variação menor que o obtido quando a barra estava a $0,3 \mathrm{~m}$ (Quadro 2).

A Figura 2 ilustra os perfis de distribuição da velocidade do vento com uma rotação do ventilador equivalente a $1.800 \mathrm{rpm}$ (a) $2.100 \mathrm{rpm}$ (b) e $2800 \mathrm{rpm}$ (c) com a barra porta-bicos a 0,3 e 0,5 m de distância.

Observa-se, na Figura 2, que os perfis de distribuição do vento são irregulares em todas as rotações e alturas da barra porta-bicos, com várias depressões ao longo de todos os perfis, reduzindo a uniformidade de distribuição do ar ao longo da barra.

\section{Uniformidade de distribuição volumétrica do conjunto de bicos hidráulicos jato cônico vazio, modelo JA-4}

Com a barra porta-bicos a $0,3 \mathrm{~m}$ do alvo, foi encontrada a seguinte relação entre as médias do coeficiente de variação da distribuição volumétrica do conjunto de bicos hidráulicos (CV) em função das rotações do ventilador (rv) e da pressão de trabalho (pt):

$$
\begin{aligned}
& C V \%)=7,24719+0,00554798^{* * *} r v+0,00281903 p t \\
& \mathrm{r}^{2}=0,9190
\end{aligned}
$$

**Significativo a $1 \%$ de probabilidade; ${ }^{\square}$ significativo a $7 \%$ de probabilidade, pelo teste " $t$ ".

Menor coeficiente de variação foi obtido com menor rotação do ventilador e menor pressão de trabalho. Com o aumento da rotação do ventilador ocorreram perfis irregulares em todas as rotações, com várias depressões na zona central, impedindo

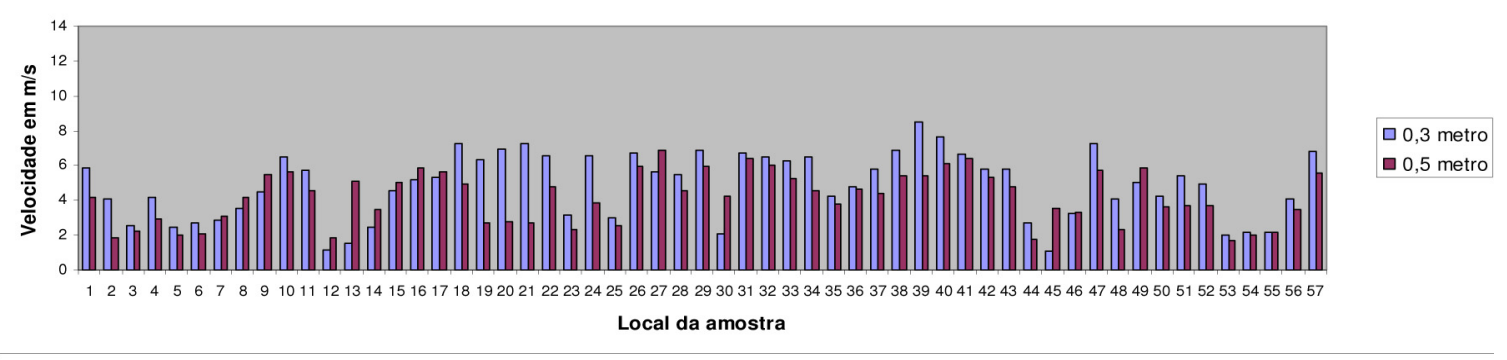

(a)

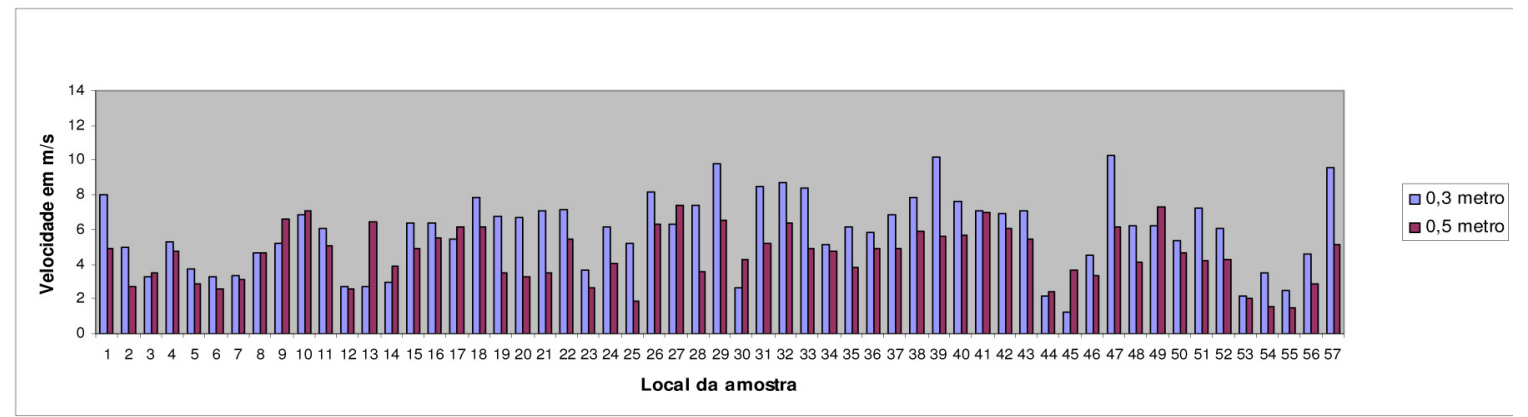

(b)

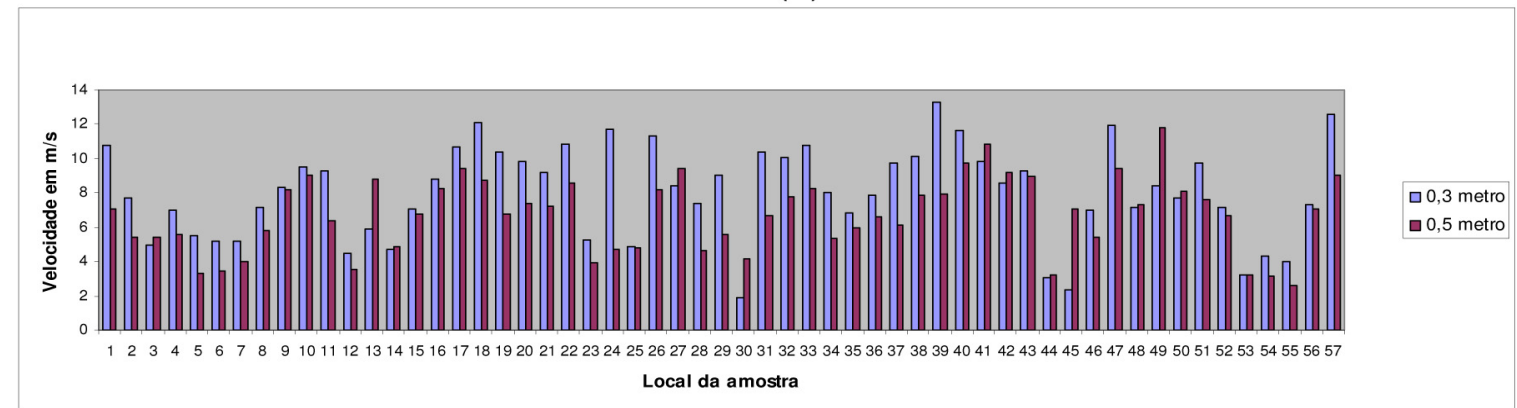

(C)

Figura 2. Perfil de distribuição da velocidade do vento trabalhando com a barra porta-bicos a 0,3 e $0,5 \mathrm{~m}$ de distância e rotação do ventilador de 1.800 (a), 2.100 (b) e 2.800 rpm (c). 
uma boa uniformidade de distribuição do tratamento em área total. Na rotação de $2.100 \mathrm{rpm}$, o coeficiente de variação estimado foi de $20,02 \%$, com uma pressão de trabalho de $400 \mathrm{kPa}$.

Com a barra porta-bicos a $0,4 \mathrm{~m}$ do alvo, as rotações do ventilador e as pressões de trabalho não influenciaram o coeficiente de variação $(\mathrm{CV})$ da distribuição volumétrica do conjunto de bicos hidráulicos, que obteve um valor médio estimado em 20,44\%. Uma distribuição volumétrica irregular pode implicar em nova infestação da área após a pulverização, além de estimular o aparecimento de patógenos mais resistentes.

Quando a barra porta-bicos estava posicionada a uma altura de $0,5 \mathrm{~m}$ do alvo, o menor coeficiente de variação foi obtido com menor rotação do ventilador e menor pressão de trabalho (Equação 4). Assim, quando a assistência de ar estava ligada, a uniformidade de distribuição foi mais comprometida, uma vez que ocorreu aumento no número de depressões centrais, agravando o problema de distribuição. Resultado semelhante ocorreu com o aumento da pressão de trabalho. Pôde-se observar, também, que na rotação de $2.100 \mathrm{rpm}$, o coeficiente de variação estimado foi de $21,28 \%$, com uma pressão de trabalho de $400 \mathrm{kPa}$.

$$
\begin{aligned}
& C V \%)=4,61+0,00154897^{* *} r v+0,0335645^{* *} p t \\
& \mathrm{r}^{2}=0,9309 .
\end{aligned}
$$

**Significativo a $1 \%$ de probabilidade, pelo teste " $\mathrm{t}$ ".

De maneira geral, os melhores resultados foram encontrados quando a barra porta-bicos estava a $0,3 \mathrm{~m}$ do alvo. Isso pode ser explicado devido à relação do ângulo de pulverização e da altura da barra em relação ao alvo, já que nessa altura não ocorreu sobreposição de um bico hidráulico sobre o outro, o que em alguns casos prejudicou o perfil de distribuição.

\section{Uniformidade de distribuição volumétrica do conjunto de bicos hidráulicos jato leque, modelo AXI-110-04}

Com a barra porta-bicos a $0,4 \mathrm{~m}$ do alvo, foi encontrada a seguinte relação entre as médias do coeficiente de variação da distribuição volumétrica do conjunto de bicos hidráulicos (CV) em função das rotações do ventilador (rv) e da pressão de trabalho (pt):

$$
\begin{aligned}
& \text { CV }(\%)=6,5985+0,001204 r v+0,001114 p t-0,000007412 * v v x p t \\
& r^{2}=0,6572
\end{aligned}
$$

*Significativo a $5 \%$ de probabilidade; ${ }^{\square}$ significativo a $7 \%$ de probabilidade; e ${ }^{\square}$ significativo a $44 \%$ de probabilidade, pelo teste " $\mathrm{t}$ ".

Com uma rotação do ventilador de $2.100 \mathrm{rpm}$, o coeficiente de variação estimado foi de 7,68; 6,24; e $4,79 \%$, com uma pressão de trabalho de 100,200 e $300 \mathrm{kPa}$, respectivamente. Assim, pode-se afirmar que o aumento na pressão de trabalho melhorou a distribuição volumétrica da calda. Fixando uma pressão de trabalho de $300 \mathrm{kPa}$, o coeficiente de variação estimado foi de 6,$93 ; 5,08 ; 4,79$; e $4,08 \%$, com uma rotação do ventilador de $0,1.800,2.100$ e $2.800 \mathrm{rpm}$, respectivamente. Assim como a pressão de trabalho, o aumento na rotação do ventilador melhorou a distribuição volumétrica da calda.

Com a barra a $0,5 \mathrm{~m}$ do alvo, menor coeficiente de variação foi obtido com menor rotação do ventilador e maior pressão de trabalho (Equação 6). Com o aumento da rotação do ventilador ocorreram perfis irregulares em todas as rotações, novamente com algumas depressões na zona central, impedindo a boa uniformidade de distribuição do tratamento em área total. Na rotação de 2.100 rpm, o coeficiente de variação estimado foi de 7,07\%, com uma pressão de trabalho de $300 \mathrm{kPa}$.

$$
\begin{aligned}
& C V(\%)=6,86536+0,000933539 r v-0,00584940^{*} p t \\
& \mathrm{r}^{2}=0,5889
\end{aligned}
$$

*Significativo a $5 \%$ de probabilidade; $\mathrm{e}^{\square}$ significativo a $7 \%$ de probabilidade, pelo teste " $\mathrm{t}$ ".

Com a barra porta-bicos a $0,6 \mathrm{~m}$ do alvo e uma rotação do ventilador de $2.100 \mathrm{rpm}$, o coeficiente de variação estimado foi de 8,$69 ; 8,19$; e 7,69\%, com uma pressão de trabalho de 100, 200 e $300 \mathrm{kPa}$, respectivamente (Equação 7). Assim, pode-se afirmar que o aumento na pressão de trabalho melhorou a distribuição volumétrica da calda. Fixando uma pressão de trabalho de $300 \mathrm{kPa}$, o coeficiente de variação estimado foi de 7,$93 ; 7,08 ; 7,69$; e $9,96 \%$ com uma rotação do ventilador de $0,1.800,2.100$ e $2.800 \mathrm{rpm}$, respectivamente, mostrando que, de maneira geral, a rotação do ventilador melhorou a distribuição volumétrica da calda.

$$
\begin{aligned}
& \text { CV } \%)=9,42673-0,00263557^{\circ} r v+0,0000012^{\prime \prime} r v^{2}-0,00497775^{\circ} p t \\
& r^{2}=0,6421
\end{aligned}
$$

** Significativo a $1 \%$ de probabilidade; *significativo a $5 \%$ de probabilidade; e ${ }^{\square}$ significativo a $10 \%$ de probabilidade, pelo teste " $\mathrm{t}$ ". 
De maneira geral, os melhores resultados foram encontrados quando a barra estava a 0,4 e $0,5 \mathrm{~m}$ do alvo. Isso pode ser explicado pela relação do ângulo de pulverização e altura da barra em relação ao alvo, já que nessa altura ocorreu sobreposição de um bico sobre o outro, o que em alguns casos pode melhorar o perfil de distribuição. Destacouse, também, o fato de o valor do coeficiente de variação obtido com o ventilador desligado não ter sido diferente de quando esse aparelho estava trabalhando.

Os resultados de laboratório evidenciaram que os bicos hidráulicos do tipo leque apresentaram os menores coeficientes de variação, indicando menor influência da assistência de ar na barra porta-bicos para esse tipo de bico hidráulico.

\section{CONCLUSÕES}

- Os perfis de distribuição do ar são irregulares em todas as rotações e alturas da barra porta-bicos estudadas.

- Maior pressão de trabalho melhorou a distribuição volumétrica de calda.

- Os melhores resultados foram encontrados quando a barra porta-bicos estava a 0,4 e $0,5 \mathrm{~m}$ do alvo para o bico do tipo leque e $0,3 \mathrm{~m}$ para o bico hidráulico de jato cone vazio.

- Os bicos hidráulicos do tipo leque apresentaram menores coeficientes de variação da distribuição volumétrica de calda que o bico hidráulico de jato cone vazio.

- A rotação do ventilador que apresentou os menores coeficientes de variação (\%) foi a de $2.100 \mathrm{rpm}$.

\section{REFERÊNCIAS BIBLIOGRÁFICAS}

ANDERSEN, P.G.; JORGENSEN, M.K.; TAYLOR, W.A. Hardi twin air assistance for field crop sprayers - The status after 10 years in use. Hardi international application technology course 2000. Taastrup, v.1, c.2, p. 138-144, 2000 .

BARTHELEMY, P.; BOISGOINTER, D.; JOUY, L.; LAJOUX, P. Choisir les outilis de pulverisation. Paris: Institut Technique des Céréales et des Fourrages, 1990. 160p.

BAUER, F.C.; RAETANO, C.G. Air-assisted sprayer for the evaluation of deposition and losses of pesticide application to soybean crop. Scientia Agrícola, v. 7, n.2, p.271-276, abr./jun. 2000.

ECS - European Committee for Standardization. Agricultural and forestry machinery Sprayers and liquid fertilizer distributors - Environmental protection - Part 2: Low crop sprayers - prEN 12761-2:1997. Brussels: CEN, 1997. $17 \mathrm{p}$.

ISO - International Organization for Standardization. Equipment for crop protection. Spraying equipment - Part 2: test methods for agricultural sprayers. Geneva, ISO, 1986. 5p. (ISO 5682/2).

MATTHEWES, G.A. Pesticide application methods. Malden: Blackwell Science, 2000. $432 p$.

MATTHEWES, G.A. How was the pesticide applied? Crop protection, v.23, n.7, p.651-653, 2004.

TEIXEIRA, M.M.; RUAS, R.A.; BAESSO, M.M.; MAGNO JUNIOR, R.G. Controle da qualidade de aplicação de fitossanitários nas propriedades agrícolas. Engenharia na Agricultura. n.11, p.29, 2008. (Boletim Técnico). 